\title{
ANÁLISIS DE LA PRÁCTICA EDUCATIVA DEL ÁMBITO DE INTERVENCIÓN DE LA FORMACIÓN BÁSICA DE JÓVENES Y ADULTOS: MÁS ALLÁ DE LO MERAMENTE ACADÉMICO
}

\author{
ANALYSIS OF THE EDUCATIONAL PRACTICE OF THE INTERVENTION \\ AREA IN THE BASIC EDUCATION OF YOUNG PEOPLE AND ADULTS: \\ BEYOND THE MERE ACADEMIC MATERS
}

\author{
$M^{a}$ Carmen Ricoy Lorenzo* y Tiberio Feliz Murias**
}

\section{RESUMEN}

En este artículo realizamos un análisis de la educación de personas jóvenes y adultas desde sus ámbitos de intervención y aproximación legislativo-curricular de la formación básica. Así mismo, recogemos una investigación empírica que nos permite analizar, contrastar y conocer la situación educativa estudiada en contextos naturales. Esta investigación se desarrolla en la Comunidad Autónoma Gallega, involucrando en la misma al alumnado y profesorado a través de una perspectiva metodológica cuantitativo-cualitativa.

Palabras clave: educación básica de jóvenes y adultos; intervención educativa; desarrollo personal.

\begin{abstract}
In this article, we carry out an analysis of the training of adult people, starting from its intervention areas, its contextual framework, and its legislative, curricular context in the basic training. Likewise, we explain a descriptive research that allows to analyse, interpret, and know the educational situation, studying it in natural contexts. This research was developed in the Spanish region of Galicia (Northwest of Spain), involving the students and teachers through a qualitative, quantitative methodology.
\end{abstract}

Key words: education of young people; adult education; educational intervention; personal development.

\footnotetext{
* Profesora Contratada Doctora en la Facultad de CC. de la Educación de la Universidad de Vigo. E-mail: cricoy@uvigo.es.

** Profesor numerario de la Facultad de Educación de la UNED. E-mail: tfeliz@edu.uned.es.
} 


\section{Introducción}

En España los antecedentes de la educación de adultos se ubican en la "Corriente Liberal Burguesa", a través de las "Sociedades Económicas del País", éstas pretenden elevar el nivel cultural del pueblo y así facilitar el crecimiento formativo (Jabonero, López, y Nieves, 1999). La organización institucional de la educación básica para personas adultas en nuestro país se producía con la Ley Moyano de 1857 y se encuentra impregnada de un matiz compensatorio. Del que no se despegará con la concepción que adopta la Ley General de Educación de 1970, la Ley de Ordenación General del Sistema Educativo (LOGSE) español de 1990 ni la reciente Ley Orgánica de Educación (LOE) de 2006.

La terminología al uso acuñada en España para dirigirnos al campo educativo que estamos tratando, y particularmente, al de la educación básica de adultos ha sido la de "Educación de Adultos". Nosotros preferimos referirnos a la educación de jóvenes y adultos atendiendo a la edad cronológica que abarca el colectivo, en vez de ceñirnos a la referida nomenclatura.

Los ámbitos de intervención en la formación de personas jóvenes y adultas se han configurando paulatinamente en las últimas décadas, contando con los numerosos cambios que se producen tanto a nivel local como mundial. Cada vez, en mayor medida, a estas personas se le presenta la necesidad de adquirir, completar y actualizar su formación en el campo de la educación formal, la no formal o la informal (Gelpi, 1989).

Coincidimos con Requejo (1997), en que los elementos clave de la educación para jóvenes y adultos están relacionados con decisiones curriculares que no son meramente técnicas. Estos integrantes de la acción educativa deben conjugar la realidad contextual (sociofamiliar, laboral, etc.) con la dimensión didáctica, psicológica y disciplinar. Por ello, las medidas curriculares a tomar no son solo técnicas o didácticas. Además, es un hecho que desde la educación básica para jóvenes y adultos se deben atender sus principales carencias de tipo sociopersonal e instrumental para que puedan desenvolverse con normalidad en la vida cotidiana (Ricoy, 2005). Ello, implicándoles en su proceso formativo para potenciar, en lo posible, la autoformación permanente.

El trabajo que aquí presentamos se enmarca en una investigación más amplia sobre la educación básica de jóvenes y adultos desarrollada en la Comunidad Autónoma Gallega. La pretensión de este estudio fue ir más allá de lo meramente académico, adentrándonos en elementos que gozan de relevancia para facilitar una educación adaptada a las necesidades, limitaciones y expectativas de las personas. La formación de este colectivo no puede entenderse sin contar con un curriculum que contemple su idiosincrasia.

El objetivo general de la investigación fue conocer las dinámicas en las que se lleva a cabo la educación básica de jóvenes y adultos, asentando el estudio sobre diversas dimensiones que nos permitieron ahondar en distintos aspectos. El propósito principal del estudio (por lo que respecta al trabajo aquí presentado) se centra en identificar los mecanismos, más allá de los elementos estrictamente curriculares, en los que se encuentra inmersa la educación básica de jóvenes y adultos. Los objetivos específicos vinculados con el general, que hemos planteado para la parte de la investigación aquí tratada son los siguientes:

- Conocer el prototipo de participación que se genera en la educación de jóvenes y adultos, para acercarnos a las dinámicas en las que se lleva a cabo su formación básica. 
- Perfilar el tipo de respuesta educativa que se contempla en el ámbito de intervención de la formación básica de personas jóvenes y adultas.

- Identificar en que medida se tienen en cuenta, más allá de lo meramente curricular, en la educación de jóvenes y adultos las necesidades, posibilidades y demandas que presentan.

- Indagar sobre el nivel de coherencia que presentan los elementos curriculares que se abordan en la educación de jóvenes y adultos atendiendo a las peculiaridades del colectivo.

\section{Marco de referencia}

En este apartado nos acercarnos al campo de la formación de personas jóvenes y adultas, contemplando someramente sus diversos ámbitos de intervención educativa, así como su aproximación legislativo-curricular.

\section{Delimitación de los ámbitos de intervención de la educación de jóvenes y adultos}

La clasificación sobre los ámbitos de intervención de la educación de jóvenes y adultos recoge grandes ejes de actuación, en torno a los que se desarrolla este campo de la formación de adultos en nuestro país. Las personas jóvenes y adultas pueden participar en uno o más ámbitos de intervención educativa, a la vez, en función de sus necesidades y preferencias vitales.

Para acotar los ámbitos de intervención en la "educación de adultos", se han trazado unas líneas básicas de actuación, en las que confluyen distintos autores (Ferrández, 1994; Medina y Gento, 1995) con las consideradas por la LOGSE de 1990, en su Título III dedicado a la educación de adultos. Estos ámbitos de intervención contemplan la adquisición y actualización de la formación, obtención o mejora de su cualificación profesional, desarrollo de la facultad de participación social, cultural, política y económica y la educación para el ocio. Con todo, la referida Ley priorizó la educación básica por estimar que su carencia se proyecta como el fundamental obstáculo para la inserción laboral. El mismo Delors (1996) entiende que la educación básica es el pasaporte indispensable para toda la vida que permite elegir y condiciona lo que se hará en el futuro en la vida laboral, sociofamiliar y recreativa.

Por su parte, los referentes barajados sobre la formación ocupacional o reciclaje profesional hacen hincapié en la obtención de la educación y actualización de cara al mundo del trabajo. Esta formación se encamina al desarrollo y mejora permanente de las personas en un contexto de cambios constantes.

En España coexisten dos subsistemas de Formación Profesional: formación profesional reglada inserta en el Sistema Educativo con carácter inicial (de $1^{\circ}$ y $2^{\circ}$ grado) y el de la ocupacional, no reglada de carácter continuo. En ambos casos, esta formación se centra en la preparación para el desarrollo de la actividad laboral y tiene lugar, para el primer caso, dentro del sistema educativo reglado y en el segundo se sitúa en el marco de la empresa encontrándose a caballo entre la formación educativa propiamente dicha y el mundo laboral (Jover, 1999). 
La educación para la participación en la vida cultural, social, económica y política, así como la educación para el ocio contribuyen al disfrute sociopersonal de la ciudadanía. Formación que se encuadra en la educación no formal e informal. Este tipo de formación adquiere un cariz más personificado, cuando se corresponde con el subsistema de educación informal posibilitando el enriquecimiento a las personas en su faceta privada. Pastor (2001) se refiere a la educación informal como aquella que influencia a los sujetos en su vida cotidiana sin producirse como actividad educativa propiamente dicha, sino presentándose de modo tan corriente como leyendo el periódico o un libro, viendo la televisión, escuchando la radio, relacionándose con los demás, jugando, etc.

\section{Aproximación legislativo-curricular a la educación básica de jóvenes y adultos}

El estudio que recogemos en este artículo se ubica en la Comunidad Autónoma Gallega, por ello nos centramos prioritariamente en el marco legislativo-curricular de la educación básica para jóvenes y adultas de dicha Comunidad.

Las enseñanzas puestas en marcha por la Ley General de Educación de 1970 para la educación básica de jóvenes y adultos terminaron recientemente su proceso de extinción en el estado español. En la actualidad se encuentra implantada la Ley de Ordenación General del Sistema Educativo de 1990 en los diferentes niveles de la educación de jóvenes y adultos y previsiblemente en el futuro se incorporarán los tímidos cambios desencadenados por la Ley Orgánica de Educación de 2006. Esta última no incorpora variaciones apreciables sobre la anterior.

Hasta el curso 2001-2002, en los centros de formación de adultos de nuestra Comunidad Autónoma se ofertan las enseñanzas conducentes a la Titulación de Graduado Escolar y las encaminadas a la obtención del Graduado en Educación Secundaria. Con la aplicación del real Decreto 1487/1994, de 1 de julio (BOE del 28), se modifica y completa el real Decreto 986/1991, de 14 de junio, aprobándose el calendario de procedimiento de la nueva ordenación del sistema educativo.

Por resolución de 14/7/1997, la Dirección General de Educación y de Formación Profesional de la Junta de Galicia convoca pruebas de madurez para adquirir el Título de Graduado Escolar para Personas Adultas (Diario Oficial de Galicia, 4/3/1998). A las pruebas de madurez para la obtención de la Titulación de Graduado Escolar podían acudir las personas mayores de 14 años (que no estuviesen siguiendo enseñanzas regladas en el sistema educativo ordinario). Los contenidos de estas pruebas, expuestos en la "Documentación sobre la implantación de la enseñanza básica para personas adultas de la Junta de Galicia" de 1997 versaban sobre las áreas de: Matemáticas y Ciencias de la Naturaleza; Ciencias Sociales; Lengua Extranjera; Lengua Castellana; y Lengua Gallega.

La situación con la que nos encontramos en la Comunidad Autónoma hace que nos remitamos a la "Ley Gallega de Educación y Promoción de Adultos" de 1992 (Ley, 9/1992, del 24 de julio, Diario Oficial de Galicia de 6 de agosto). Esta Ley es el marco legal, al amparo del cual, la Administración Educativa y los centros en donde se aborda la formación de jóvenes y adultos se acogen. La implantación de Ley de Educación de Adultos en Galicia se hizo esperar, fue con la orden 26/5 de 1997 (Diario Oficial de Galicia de 15/7/97) en la que se regula la enseñanza de base para adultos, estableciendo su estructura y curriculum de la Comunidad Autónoma. 
La investigación abordada se centra en la educación de base, en los niveles que se corresponden con las Titulaciones de Graduado Escolar (ya comentado) y con la de Graduado en Educación Secundaria (nivel III). Los campos de conocimiento del curriculum de Educación Secundaria para jóvenes y adultos en Galicia son: Sociedad (CC. Sociales, Geográfica e Historia, Educación Plástica y Visual y Música); Naturaleza (CC. Naturales y de Educación Física); Comunicación (Lengua y Literatura Gallega y Castellana, Lenguas Extranjeras y contenidos sobre comunicación en Educación Plástica y Visual y Música); TecnológicoMatemático (Matemáticas, Tecnología y Educación Plástica y Visual).

La Junta de Galicia regula las enseñanzas de base para personas jóvenes y adultas, estableciendo su estructura y currículum en esta Comunidad, en la Orden de 26/5/1997 (Diario Oficial de Galicia de 15/7/1997). Para obtener el Título de Graduado en Educación Secundaria, los mayores de 18 años cuentan además de las posibilidades expuestas, con la medida recogida en el Decreto 315/1996 de 26 de julio para la realización de pruebas periódicas con carácter libre (Diario Oficial de Galicia, 19/5/1997).

\section{Método}

El diseño utilizado en esta investigación combina el corte transversal con el longitudinal y nos permitió reunir información en momentos puntuales (a través del cuestionario) y también a lo largo de un amplio periodo (con la aplicación de entrevistas). Siguiendo a Feliz y Ricoy (2003) la propia realidad está configurada por elementos cualitativos y cuantitativos, por lo que resultaba adecuada una aplicación bimetódica en su desarrollo.

El estudio se sitúa en el enfoque de investigación descriptivo-interpretativo en el que abordamos diferentes dimensiones y utilizamos distintos instrumentos de recogida de información. Los datos cuantitativos recogidos fueron útiles para estudiar gran cantidad de sujetos y los cualitativos para concentrarnos en un mayor nivel de profundización, atendiendo a un número reducido. Esta investigación posibilitó establecer relaciones y contrastar los resultados complementándose entre sí (Cohen y Manhion, 1990).

\section{Muestra}

Por lo que respecta al cuestionario, la muestra fue obtenida por muestreo no-probabilístico, al seleccionarla de este modo tuvimos en cuenta elementos y criterios que nos permitían garantizar la representatividad (Tashakkori y Teddlie, 1998). Invitamos a participar en la investigación a todo el alumnado y profesorado de la Comunidad Autónoma, que se encontraba en la educación del último nivel conducente a la Titulación de base. Contamos con la colaboración de los que recibían formación presencial y a distancia, así como con los que se encontraban ubicados en el entorno urbano y el rural.

A partir de la cumplimentación del cuestionario del alumnado se originaron datos de 512 sujetos (50,2\% mujeres y 49,8\% varones) de 24 centros educativos, mientras que el del profesorado aportó información de 110 docentes (68,2\% mujeres y $31,8 \%$ varones) de 26 centros. La totalidad de personas involucradas en la cobertura de los cuestionarios suponen una participación de 622 sujetos. Así mismo, hemos tenido en cuenta para el muestreo el carácter público del centro (Centros Públicos de Educación Permanente de Adultos e Insti- 
tutos de Educación Secundaria) y no incluimos los privados dada la escasa presencia de éstos o inexistencia en la mayoría de las provincias de la Comunidad Autónoma Gallega.

Para realizar las entrevistas hicimos una selección eligiendo "personas clave" entre el alumnado y profesorado que más credibilidad nos ofrecía como informante considerando, además, su accesibilidad. En la elección de estos participantes tuvimos en cuenta diferentes variables: edad, género, procedencia, años de experiencia en la formación de adultos, (en el caso del profesorado), etc.

El número de entrevistados fue determinado a lo largo de su desarrollo en función del nivel de saturación de los datos. En total fue necesario entrevistar a 16 sujetos en el caso del alumnado (6 alumnas y 10 alumnos) y 20 en el caso del profesorado (12 mujeres y 8 varones).

\section{Análisis aplicados}

Los datos de carácter cuantitativo del cuestionario los hemos tratado con el paquete de análisis estadístico para las Ciencias Sociales SPSS, versión 10.0 para Windows. En el trabajo aquí presentado aportamos resultados de la investigación que nos posibilitan contrastar los resultados obtenidos con el alumnado y profesorado.

Para confrontar la información recogida con los ítems del cuestionario de discentes y docentes hemos aplicado el análisis de varianza de una sola vía. De estos análisis seleccionamos para el presente artículo los resultados de diferentes estadísticos (Media Aritmética, Desviación Típica, Grado de Libertad, Ratio (F) y significatividad como exponemos más adelante.

Los análisis de la información obtenida mediante la entrevista fueron realizados con el programa AQD (Analysis of Qualitative Data) versión 6.0 para Windows. Este programa de estudio cualitativo nos permite abordar en profundidad los análisis de contenido de las entrevistas (Huber, 2003).

Las distintas categorías generales y específicas (referidas a cada una de las preguntas de la entrevista) las creamos a partir de sus transcripciones. Este programa informático posibilita incorporar a los datos de naturaleza cualitativa sus frecuencias y porcentajes respectivos.

\section{Resultados}

En el presente trabajo recogemos los resultados de ítems principales de los cuestionarios y preguntas clave de las entrevistas. Estos datos nos posibilitan encontrar los paralelismos y discrepancias asumidas por ambos colectivos a partir de la información recogida con sendos instrumentos.

\section{Cuestionario}

De los resultados estadísticos obtenidos a partir del cuestionario procedentes del análisis de varianza presentamos en las tablas 1 y 2 respectivamente la Media Aritmética, Desvia- 
TABLA 1: Aspectos que contemplan en la educación de jóvenes y adultos.

\begin{tabular}{|c|c|c|c|c|c|c|c|}
\hline \multirow[b]{2}{*}{ Variable dependiente } & \multicolumn{2}{|c|}{$\mathbf{N}$} & \multirow[b]{2}{*}{$\mathbf{X}$} & \multirow[b]{2}{*}{$\mathbf{S}$} & \multirow[b]{2}{*}{ G.L. } & \multirow[b]{2}{*}{$\mathbf{F}$} & \multirow[b]{2}{*}{ Sig. } \\
\hline & $\begin{array}{l}\text { Alum- } \\
\text { nado }\end{array}$ & $\begin{array}{l}\text { Profe- } \\
\text { sorado }\end{array}$ & & & & & \\
\hline $\begin{array}{l}\text { Concretan conjuntamente las } \\
\text { necesidades de formación del grupo }\end{array}$ & \multirow{3}{*}{512} & \multirow{3}{*}{110} & $\begin{array}{l}3,4785 \\
3,5963\end{array}$ & $\begin{array}{l}1,2850 \\
1,0639\end{array}$ & \multirow{4}{*}{1,620} & 0,7993 & 0,3716 \\
\hline Entablan retos formativos & & & $\begin{array}{l}3,2227 \\
3,3486\end{array}$ & $\begin{array}{l}1,2248 \\
1,0833\end{array}$ & & 0,9881 & 0,3206 \\
\hline $\begin{array}{l}\text { Involucran en experiencias que } \\
\text { potencian el autoaprendizaje }\end{array}$ & & & $\begin{array}{l}3,7363 \\
3,8364\end{array}$ & $\begin{array}{l}1,1480 \\
0,9817\end{array}$ & & 0,7217 & 0,3959 \\
\hline $\begin{array}{l}\text { Desarrollan habilidades que } \\
\text { mejoran las relaciones sociales }\end{array}$ & \multicolumn{2}{|c|}{ Total: 622} & $\begin{array}{l}3,3275 \\
3,6091\end{array}$ & $\begin{array}{l}1,2864 \\
1,0501\end{array}$ & & 4,6083 & 0,0322 \\
\hline
\end{tabular}

TABLA 2: Interés manifestado por el desarrollo de la clase.

\begin{tabular}{|c|c|c|c|c|c|c|c|}
\hline \multirow{2}{*}{ Variable dependiente } & \multicolumn{2}{|c|}{$\mathbf{N}$} & \multirow{2}{*}{$\mathbf{X}$} & \multirow{2}{*}{$\mathbf{S}$} & \multirow{2}{*}{ G.L. } & \multirow{2}{*}{$\mathbf{F}$} & \multirow{2}{*}{ Sig. } \\
\hline & Alumnado & Profesorado & & & & & \\
\hline E los temas que tratan & \multirow{3}{*}{512} & \multirow{3}{*}{110} & $\begin{array}{l}3,8477 \\
3,3545\end{array}$ & $\begin{array}{l}1,0927 \\
0,8193\end{array}$ & \multirow{4}{*}{1,620} & 19,9778 & 0,0000 \\
\hline $\begin{array}{l}\text { Las actividades o } \\
\text { ejercicios que realizan }\end{array}$ & & & $\begin{array}{l}3,8652 \\
3,5273\end{array}$ & $\begin{array}{l}1,0905 \\
0,9258\end{array}$ & & 9,1455 & 0,0026 \\
\hline $\begin{array}{l}\text { Las explicaciones que } \\
\text { se dan en la clase }\end{array}$ & & & $\begin{array}{l}4,0762 \\
3,5545\end{array}$ & $\begin{array}{l}1,1121 \\
0,9344\end{array}$ & & 21,0053 & 0,0000 \\
\hline $\begin{array}{l}\text { La participación que } \\
\text { se genera }\end{array}$ & \multicolumn{2}{|c|}{ Total: 622} & $\begin{array}{l}3,6118 \\
3,3909\end{array}$ & $\begin{array}{l}1,1653 \\
1,0413\end{array}$ & & 3,3702 & 0,0669 \\
\hline
\end{tabular}

ción Típica, Grado de Libertad, Ratio (F) y Significatividad o Probabilidad (para un nivel de confianza del 95\% con una significatividad estadística de 0,05).

Constatamos (tabla 1) que existe una elevada sintonía entre las respuestas proporcionadas por el alumnado y el profesorado sobre diversos aspectos planteados (variable dependiente). Más allá de lo estrictamente académico se contempla en la educación de jóvenes y adultos la: concreción de las necesidades de formación que presentan los destinatarios; impulso de retos formativos; implicación en experiencias que potencian el autoaprendizaje permanente; y el compromiso en el desarrollo de habilidades que optimizan las relaciones sociolaborales.

En cuanto a la percepción que tienen ambos colectivos sobre el desenvolvimiento de habilidades que mejoran las relaciones sociolaborales, se presenta diferencias significativas en esta variable con un nivel de significación estadística de 0,05 (n.c. del 95\%).

El grado de consideración que muestra el alumnado y profesorado sobre distintos elementos más allá de lo curricular, en la educación de jóvenes y adultos se encuentra en una 
situación dialógica aceptable. Desde este planteamiento se trata de adquirir a partir de la contextualización e idiosincrasia que presenta el grupo de alumnos del aula la formación más idónea que precisan para desarrollarse en la vida. Compartimos con Ortiz (2002), la idea de tratar de que los jóvenes y adultos en su educación básica identifiquen, comprendan y acepten lo que le es necesario aprender desde un contexto democrático de responsabilidad compartida. No podemos obviar, que la formación básica es un peldaño que facilita la entrada en el mundo laboral y sociorrelacional o puede mejorarla.

Detectamos (tabla 2) un interés notorio por parte del alumnado y profesorado en las dinámicas en las que se encuentran inmersos en el proceso de enseñanza o aprendizaje (variable dependiente). El alumnado y los formadores implicados en la educación de jóvenes y adultos concuerdan en la existencia del cálido clima de intervención en que se desarrolla la clase, el interés existente por los contenidos temáticos que tratan y las explicaciones proporcionadas en el aula, así como por las actividades o ejercicios que realizan. Encontrando que la visión sobre los referidos hechos que tiene el alumnado es ligeramente más optimista que la que presenta el profesorado.

Hemos de reseñar que el contraste entre los resultados obtenidos en las 3 primeras variables a partir de las consideraciones que tiene el alumnado y profesorado, ponen de manifiesto que existen diferencias estadísticamente significativas (n.c. del 95\% y significatividad estadística del 0,05) sobre: el interés plasmado por los contenidos temáticos, la realización de actividades y las explicaciones facilitadas en la clase. Con todo, en los tres casos y en ambos colectivos el interés emitido en cuanto a los aspectos antes referidos es favorable.

Es un hecho que la educación de jóvenes y adultos demanda dinámicas de acción acordes con este colectivo, que deben atender de manera acorde las peculiaridades y necesidades que presenta. Ricoy (2004) apunta que la respuesta educativa para jóvenes y adultos en la formación básica debe apartarse de los modelos escolares en el diseño y desarrollo curricular. Ello, desde un elevado grado de flexibilidad e interacción con el grupo-aula, el uso de estrategias de intervención psicosocioeducativas en su doble vertiente teórico-práctica acordes con la singularidad del colectivo y la potenciación de procesos de reflexión y análisis respetuosos con la práctica formativa abordada. En esta línea, Gento (1997) afirma que la formación de este conjunto debe responder en cada caso al contexto, recursos con los que se cuente y sobre todo contemplar las demandas de estos jóvenes y adultos objeto de la acción formativa.

\section{Entrevista}

\section{Alumnado}

Comenzamos aportando los resultados que obtenemos en las entrevistas realizadas a los jóvenes y adultos que acuden a la educación básica.

El alumnado entrevistado (tabla 3) declara que en la formación de jóvenes y adultos se atiende a la: adquisición de contenidos prácticos que les facilitan desenvolverse en la vida cotidiana (apuntan argumentos al respecto 10 de los 16 entrevistados). La consideración de contenidos de carácter prácticos es un aspecto importante que pasa antes por la comprensión y estudio de lo próximo, desde la propia experiencia, para realizar posteriormente la 
TABLA 3: Aspectos que contemplan en la formación de jóvenes y adultos (alumnado).

\begin{tabular}{|c|c|c|c|c|c|c|c|c|c|c|c|c|c|c|c|c|c|c|}
\hline \multirow{2}{*}{ Categoría específica } & \multicolumn{16}{|c|}{ Número de entrevista } & \multirow{2}{*}{$\Sigma$} & \multirow{2}{*}{$\%$} \\
\hline & 1 & 2 & 3 & 4 & 5 & 6 & 7 & 8 & 9 & 10 & 11 & 12 & 13 & 14 & 15 & 16 & & \\
\hline Comunicación y trato & 1 & 0 & 1 & 1 & 0 & 1 & 1 & 0 & 0 & 1 & 0 & 1 & 0 & 0 & 0 & 1 & 8 & 34,8 \\
\hline Adquisición conten. práctic. & 0 & 1 & 1 & 1 & 0 & 1 & 1 & 1 & 0 & 0 & 1 & 1 & 0 & 1 & 1 & 0 & 10 & 43,5 \\
\hline Metodología & 0 & 0 & 0 & 0 & 1 & 1 & 0 & 0 & 1 & 0 & 0 & 1 & 1 & 0 & 0 & 0 & 5 & 21,7 \\
\hline Categoría General: Aspecto & 1 & 1 & 2 & 2 & 1 & 3 & 2 & 1 & 1 & 1 & 1 & 3 & 1 & 1 & 1 & 1 & 23 & 100 \\
\hline
\end{tabular}

transferencia más abstracta o de carácter científico. No olvidemos que este tipo de contenidos prácticos facilitan al joven y adulto el desarrollo de habilidades y destrezas útiles para su vida diaria.

El clima de aula se enriquece entablando una comunicación interactiva entre los protagonistas, desde el trato cercano entre alumnado y profesorado en el grupo-aula (exponen 8 razones). Así mismo, se utiliza una metodología activo-participativa coherente con el tipo de destinatarios, que propicia la cercanía entre éstos y atiende a sus peculiaridades (reúnen 5 argumentos al respecto). En este sentido, Sarramona (1989) indica que las directrices metodológicas en la educación de adultos deben atender a la: personalización del aprendizaje, su diversidad (personal, familiar, profesional, multicultural, etc.), empleo de recursos pertinentes y temporalización que se necesite; uso de estrategias indagativas desde planteamientos colaborativos; posibilidades y demandas que presente el colectivo conjugándolas armónicamente. En definitiva, se trata de descubrir la educación como elemento motivador de autosuperación permanente.

\section{Profesorado}

Los resultados que obtenemos a partir de las conversaciones desarrolladas en las entrevistas con el profesorado que se dedica a la educación básica de jóvenes y adultos ayudan a profundizar en la temática objeto de estudio acrecentando la visión sobre los hechos estudiados.

El profesorado entrevistado (tabla 4) entiende que incluye en la formación de jóvenes y adultos, además, de los aspectos apuntados por el alumnado otros componentes como la temporalización y la potenciación de actitudes y conductas hacia el estudio. Estos y otros elementos forman parte de la acción educativa.

El profesorado de la educación básica entrevistado reconoce que prima en la formación el uso de una metodología activo-participativa (manifestando 14 sujetos, 16 argumentos). Los formadores recalcan la relevancia que tiene el desarrollo de una comunicación plena entre los protagonistas y un buen grado de empatía personal (lo declaran 7 de los entrevistados).

Además, los docentes conceden importancia a la adquisición de contenidos de carácter práctico, trabajando a partir de la propia experiencia del alumnado (lo reconocen 4 de los entrevistados). Por último, algún educador incide también en la potenciación de actitudes y conductas que facilitan el estudio (lo apuntan 2 de los preguntados) y la cantidad y calidad del tiempo dedicado a la formación (confirmándolo un entrevistado). 
TABLA 4: Aspectos que contemplan en la formación de jóvenes y adultos (profesorado).

\begin{tabular}{|c|c|c|c|c|c|c|c|c|c|c|c|c|c|c|c|c|c|c|c|c|c|c|}
\hline \multirow{2}{*}{ Categoría específica } & \multicolumn{20}{|c|}{ Número de entrevista } & \multirow{2}{*}{$\Sigma$} & \multirow{2}{*}{$\%$} \\
\hline & 1 & 2 & 3 & 4 & 5 & 6 & 7 & 8 & 9 & 10 & 11 & 12 & 13 & 14 & 15 & 16 & 17 & 18 & 19 & 20 & & \\
\hline Potenciación act. y conductas & 0 & 0 & 0 & 0 & 0 & 0 & 0 & 0 & 0 & 0 & 0 & 0 & 0 & 1 & 0 & 0 & 0 & 1 & 0 & 0 & 2 & 6,3 \\
\hline Comunicación y trato & 0 & 1 & 1 & 0 & 0 & 1 & 0 & 0 & 1 & 0 & 1 & 1 & 0 & 0 & 0 & 0 & 0 & 0 & 0 & 1 & 7 & 21,9 \\
\hline Adquisición conten. prácticos & 0 & 0 & 0 & 0 & 1 & 0 & 0 & 1 & 0 & 0 & 0 & 0 & 1 & 0 & 1 & 0 & 0 & 0 & 0 & 0 & 4 & 12,4 \\
\hline Metodología & 2 & 1 & 1 & 1 & 0 & 1 & 2 & 1 & 1 & 1 & 1 & 1 & 0 & 0 & 0 & 1 & 1 & 0 & 1 & 0 & 16 & 50 \\
\hline Temporalización & 0 & 0 & 0 & 0 & 0 & 0 & 0 & 0 & 0 & 0 & 0 & 0 & 0 & 0 & 0 & 0 & 0 & 0 & 0 & 1 & 1 & 3,1 \\
\hline Todos & 0 & 0 & 0 & 0 & 0 & 1 & 0 & 0 & 0 & 1 & 0 & 0 & 0 & 0 & 0 & 0 & 0 & 0 & 0 & 0 & 2 & 6,3 \\
\hline Categoría General: Aspecto & 2 & 2 & 2 & 1 & 1 & 3 & 2 & 2 & 2 & 2 & 2 & 2 & 1 & 1 & 1 & 1 & 1 & 1 & 1 & 2 & 32 & 100 \\
\hline
\end{tabular}

\section{Alumnado}

Un aspecto más específico sobre el que hemos querido indagar en la educación de jóvenes y adultos se centró en el grado y tipo de participación que se genera en la clase (gráfico 1).

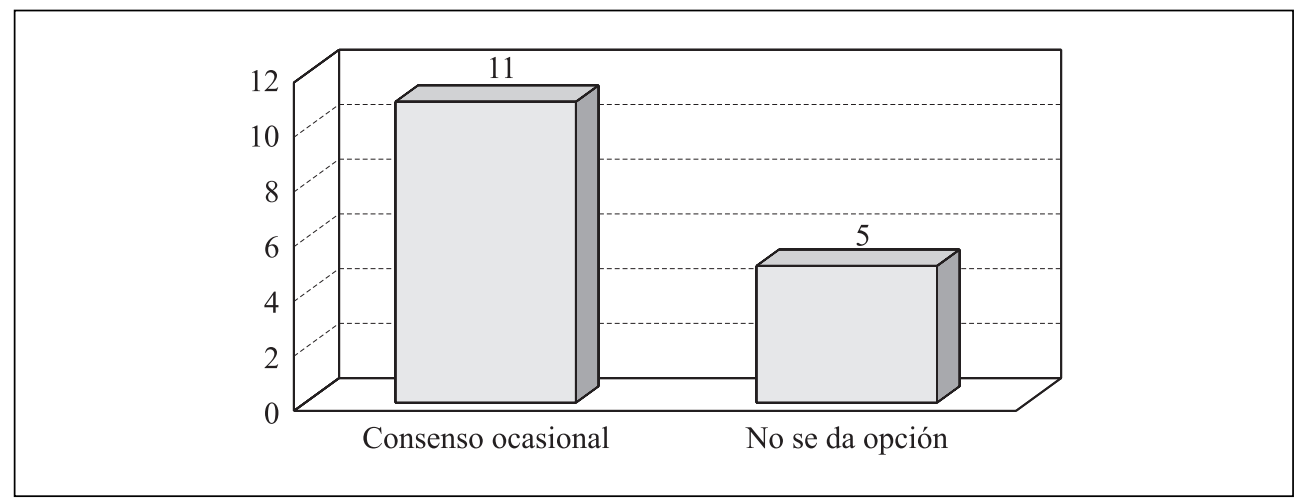

GRÁFICO 1.

Participación en la marcha de la clase (alumnado).

Una parte del alumnado joven y adulto entrevistado (11 sujetos) sostiene que su participación en el aula se da a través del consenso ocasional en algunos aspectos que afectan al desarrollo de la clase: establecimiento del horario de aula definitivo, forma de plantear la clase, temas preferentes y actividades a realizar. Otro grupo de sujetos entrevistados (5 personas), manifiesta que no se les permite la colaboración en las decisiones estrictamente curriculares (objetivos de aprendizaje, evaluación, etc.) que afectan a su formación.

En la línea de lo analizado, Leirman (1994) ha apostado por un proyecto en la educación de adultos integrando la metodología participativa, entendiendo la relevancia que tiene la interactividad continua entre los sujetos para la potenciación y desarrollo de su aprendizaje. Próxima a la directriz anterior, encontramos que Arnold (1997) defiende la importancia del aprendizaje desde la implicación de las personas jóvenes y adultas, apoyándose en el enun- 
ciado reflexivo de las demandas de formación que manifiestan. Para ello, el profesorado ayudará a estos jóvenes y adultos a asumir progresivamente un papel cada vez más activo que permita alcanzar conjuntamente las metas acordadas.

\section{Profesorado}

Los formadores de la educación de jóvenes y adultos coinciden en buena medida con lo expresado por el alumnado (gráfico 2).

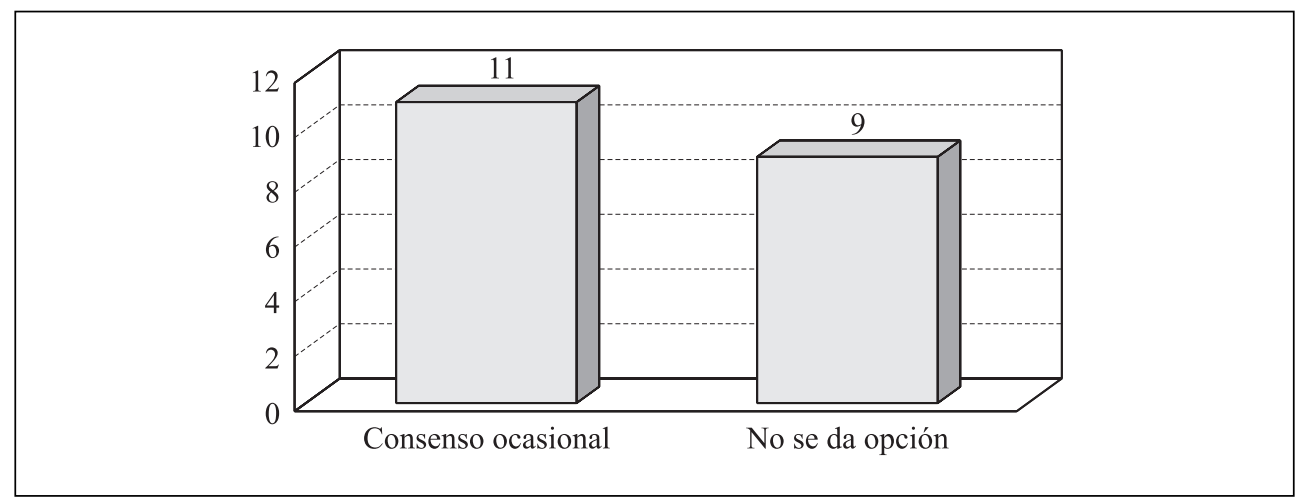

GRÁFICO 2.

Participación en la marcha de la clase (profesorado).

Parte del profesorado declara que le facilita la participación de modo esporádico en la clase al alumnado joven y adulto abarcando la negociación de aspectos metodológicos, temas a tratar, actividades a realizar y acuerdos sobre los horarios de clase (11 sujetos).

Un grupo de formadores entrevistado (9 sujetos), asegura que no le dan opción a participar en la metodología de la clase al alumnado joven y adulto. Los docentes reconocen que permiten de modo insuficiente la colaboración al alumnado, en particular cuando se trata de decisiones que afectan a los aspectos propiamente curriculares. Atribuyendo su actuación a los problemas de comportamiento que genera en la clase el alumnado menor de 20 años.

En el marco de un curriculum abierto y flexible como el que proclamó la LOGSE se ha de potenciar, en mayor medida, la participación del alumnado joven y adulto en el desarrollo de la clase. Flecha (1994) sostiene que entre los principios básicos a contemplar en la educación básica del colectivo referido está el aprendizaje significativo, partiendo del hecho de que cada uno construye la realidad atribuyéndole significados y para eso el sujeto debe estar motivado y participar activamente en su formación.

\section{Conclusiones}

Estas conclusiones están ligadas a la educación de jóvenes y adultos y son consecuencia del trabajo aquí presentado. Son un referente de partida para conocer la práctica educativa, 
desde el ámbito de intervención de la educación básica de jóvenes y adultos, más allá de lo meramente académico. El escenario analizado ofrece aportaciones de carácter situacional, ubicándose también en un marco más amplio susceptible de generalización de conocimiento (Brown y Yule, 1998; Van Zanten, 2004).

\section{Como conclusiones finales aportamos las siguientes:}

La educación de personas jóvenes y adultas se desarrolla en un clima de participación y tolerancia entre el profesorado y alumnado en cuanto a las intervenciones que se desarrollan de modo puntual e irregular en el aula. Sin embargo, subsiste el interés por abarcar las necesidades y expectativas del grupo. Este hecho, suponen un desafío importante para formadores y formandos alentándoles a asumir relaciones, compromisos y responsabilidades equilibradas, sin que esto signifique que cada colectivo altere en lo esencial su papel como educadores y educandos.

Los formadores se reservan la toma de decisión en los aspectos que tradicionalmente han sido considerados como competencia exclusiva del profesorado, referidos a los aspectos propiamente curriculares (diseño y desarrollo del programa). Lo dicho, nos hace pensar en la conveniencia de analizar las líneas de desarrollo profesional del profesorado para avanzar hacia un modelo de enseñanza colaborativo que permita mediante el trabajo en equipo de formadores y formandos precisar las prioridades y aspectos esenciales desde los que formar/se (Medina y Domínguez, 1995). Compartir el proceso de enseñanza-aprendizaje significa participar de las responsabilidades y compromisos, así como de su diseño, desarrollo y resultados.

Es necesario, además de concebir el proceso de enseñanza-aprendizaje en la educación de jóvenes y adultos como un proyecto compartido por todos sus protagonistas ligarlo al contexto mediato y lejano (Jabonero, 1997). En esta dirección, ha de continuar avanzándose hacia la consecución de un mayor equilibrio del protagonismo del alumnado en lo curricular y extracurricular.

Las dinámicas de actuación en la educación de adultos se dirigen a conocimientos de naturaleza práctica, posibilitando la interacción directa en la resolución de situaciones cotidianas. No obstante, no se orientan suficientemente a la actualización y desarrollo de habilidades que impulsen y mejoren la vida sociolaboral de las personas adultas (Clemente, 1996). En consecuencia, los diferentes diseños curriculares desde el $1^{\circ}$ hasta el $3^{\circ}$ nivel de concreción curricular deberán redefinirse, introduciendo modificaciones significativas que se plasmen en el desarrollo de la práctica educativa, con el objeto de tender puentes entre la formación, el trabajo y la vida en general (Jover, 1999). En todo caso, esto se hará concretando oportunamente las necesidades vitales que presentan las personas jóvenes y adultas para que su formación les posibilite una mejor proyección en todas sus ramificaciones.

Debe evolucionarse en el futuro en la configuración de un curriculum para jóvenes y adultos que integre pertinentemente los aspectos extracurriculares de relevancia que afectan a la vida de estas personas. Para ello, este curriculum tiene que pensarse desde una visión plenamente práctica, que permita a jóvenes y adultos formarse y madurar desde el punto de vista existencial para afrontar sus retos sociopersonales, ocupacionales y culturales de modo contextualizado dentro de un mundo cada vez más globalizado y cambiante. 


\section{Referencias bibliográficas}

Arnold, R. (1997). "Nuevas tendencias didácticas de la educación de adultos". En Actas del XI Congreso Nacional de Pedagogía (pp. 87-102). Donostia: Gipuzkoako Foru Aldundia-Diputación. Tomo III.

Brown, G. y Yule, G. (1998). Discourse analysis. Cambridge: University Press.

Clemente, A. (1996). Psicología del desarrollo adulto. Madrid: Narcea.

Cohen, L y Manhion, L. (1990). Métodos de investigación educativa. Madrid: Muralla.

Delors, J. (1996). La Educación encierra un tesoro. Madrid: Santillana.

Feliz, T. y Ricoy, M. C. (2003). "El descubrimiento de la dimensión cualitativa de la investigación a través de un foro educativo". En A. Medina y S. Castillo (Coords.), Metodología para la realización de proyectos de investigación y tesis doctorales (pp. 131-165). Madrid: Universitas.

Ferrández, A. (1994). "Educación de adultos e mundo laboral. A formación ocupacional". En A. Requejo, A. González y J. R. Lete (Coords), Educación de adultos nunha sociedade en transformación (pp. 73-85). Pontevedra: Consejo Escolar de Galicia.

Flecha, R. (1994). "Habelencias básicas da población adulta”. En A. Requejo, A. González y J. R. Lete (Coords), Educación de adultos nunha sociedade en transformación (pp. 55-71). Pontevedra: Consejo Escolar de Galicia.

Gelpi, E. (1989). "Reflexiones sobre políticas y actividades de educación permanente: historia y perspectivas". En A. Ferrández y Peiro, J. (Dirs.), Métodos y técnicas en la educación de adultos (pp. 27-36). Humanitas: Barcelona.

Gento, S. (1997). "La organización de centros y el curriculum de educación de personas adultas". En A. Medina (Coord.), Diseño y desarrollo curricular para la formación de las personas adultas (pp. 185-203). Madrid: UNED.

Huber, G. L. (2003). "Introducción al análisis de datos cualitativos". En A. Medina y S. Castillo (Eds.), Metodología para la realización de proyectos de investigación y tesis doctorales (pp. 91129). Madrid: Universitas.

Jabonero, M. (1997). "La educación de personas adultas en España: desarrollo y aplicación de la LOGSE". En A. Medina (Coord.), Diseño y desarrollo curricular para la formación de las personas adultas (pp. 19-28). Madrid: UNED.

Jabonero, M., López, I. y Nieves, R. (1999). Formación de adultos. Madrid: Síntesis.

Jover, D. (1999). "Formación de colectivos desfavorecidos como instrumento para la inserción sociolaboral". Diálogos, 17, 33-48.

Leirman, W. (1994). "O futuro da educación de adultos en Europa: Un proxecto de investigación de tipo Delphi en 14 países". En A. Requejo, A. González y J. R. Lete (Coords), Educación de adultos nunha sociedade en transformación (pp. 87-102). Pontevedra: Consejo Escolar de Galicia.

LOE (2006). Ley Orgánica 2/2006, de 3 de mayo, de educación (BOE, 106, de 4 de mayo).

LOGSE (1990). Ley Orgánica 1/1990, de 3 de Octubre, de Ordenación General del Sistema Educativo (BOE, 238, de 4 de octubre).

Medina, A. y Domínguez, C. (1995). Enseñanza y curriculum, para la formación de Personas Adultas. Madrid: EDIP.

Medina, A. y Gento, S. (1995). "Configuración del proyecto de diseño curricular en educación de adultos". En A. Medina, C. Alonso, C. Domínguez y otros (Coords.), Formación de educadores de personas adultas I (pp. 39-113). Madrid: UNED. 
Ortiz, A. (2002). "Estudio y análisis de las claves que identifican la formación básica de personas adultas en Andalucía". Enseñanza, 20, 213-239.

Pastor, M. I. (2001). "Orígenes y evolución del concepto de educación no formal”. Revista Española de Pedagogía, 220, 525-544.

Requejo, A. (1997). "Los currícula de la educación de personas adultas en el estado de las autonomías". En J. Cabello (Coord.), Didáctica y educación de personas adultas (pp. 85-104). Málaga: Aljibe.

Ricoy, M. C. (2004). "Los modelos de formación como medios para contribuir al desarrollo de las múltiples dimensiones de las personas adultas". En J. Cardona (Dir.), La calidad territorial como estrategia: La formación clave en el desarrollo (pp. 127-143). Talavera de la Reina: C.A. UNED.

Ricoy, M. C. (2005). "La prensa como recurso educativo: complejidad y pertinencia de su uso en la educación de adultos". Revista Mexicana de Investigación Educativa, 10 (2), 125-163.

Sarramona, J. (1989). "Educación de adultos por metodología no formal”. En A. Ferrández y J. Peiro (Dir.), Modelos formales y no formales en la educación de adultos (pp. 31-41). Barcelona: Humanitas.

Tashakkori, A. y Teddlie, C. (1998). Mixed methodology: Combining qualitative and quantitative approaches. Thousand Oaks, CA: Sage.

Van Zanten, A. (2004). "Pesquisa qualitativa em educaçao: pertinencia, validez e generalizaçao". Perspectiva, 22 (1), 25-45.

Fecha de recepción: 09-05-05

Fecha de revisión: 27-09-06

Fecha de aceptación: 17-10-06 